\title{
XVI.
}

Aus dem physiologischen Institut zu Leipzig.

\section{Eine neue Methode \\ zur Gewinnung von Corrosionspräparaten des Ohrlabyrinths.}

Von

\author{
Dr. 0. Eichler.
}

(Hierzu 2 Abbildungen.)

(Von der Redaction übernommen am 27. Juni 1890.)

Durch Herrn Prof. Ludwig, welcher mich im Sommer 1889 auf die Abhandlung von Siebenmann "Ueber die Injection der Knochenkanäle des Aquaeductus vestibuli et cochleae mit Woodschem Metall" aufmerksam machte, wurde ich veranlasst, mich näher mit der Corrosionsanatomie des Ohrlabyrinths zu beschäftigen. Hierbei bemerkte ich, dass die bisherigen Verfahrungsarten in mehr als einer Hinsicht unvollkommen waren. Deshalb bin ich an die Bearbeitung des Gegenstandes mit einer neuen, im Folgenden zu beschreibenden Methode herangetreten.

Das seiner Weichtheile beraubte Labyrinth bildet einen mit Luft erfüllten Raum. Soll dieser mit irgend einer Masse vollständig injicirt werden, so müssen im Allgemeinen folgende Bedingungen erfüllt werden:

1. das Labyrinth muss gut macerirt und getrocknet sein;

2. sämmtliche Oeffnungen des Knochens müssen luftdicht verschlossen werden mit Ausnahme der Eingussöffnung und einer Oeffnung zum Entweichen der Luft. Die Wabl der Eingussöffnung wird von folgender Ueberlegung bestimmt: Handelt es sich um die Darstellung des Labyrinths ohne Beriucksichtigung des ovalen Fensters, so ist dieses die bequemste Eingussöffnung; soll dagegen besonders die Form der Fenster gewonnen werden, so ist auch die Fenestra ovalis zu schliessen und ein Bobrloch im Promontorium als Eingussöffnung zu benutzen;

3. das Labyrinth muss luftleer gemacht werden. 
Methode zur Gewinnung von Corrosionspräparaten des Obrlabyrinths. 199

Hier entsteht die Aufgabe, einerseits die Luft zu entfernen, andererseits dafür zu sorgen, dass während des Eingiessens der Injectionsmasse weder die entfernte Luft, noch die atmosphärische wieder Zutritt gewinnt. Die bisherigen Verfahren suchten die Entfernung der Luft durch längeres Erwärmen des Knochens zu bewerkstelligen und das Wiedereindringen der Luft durch einen um das Präparat gelegten Gypsmantel zu verhindern. Wegen der Porosität dieses Materials leuchtet ein, wie unsicher diese Me-

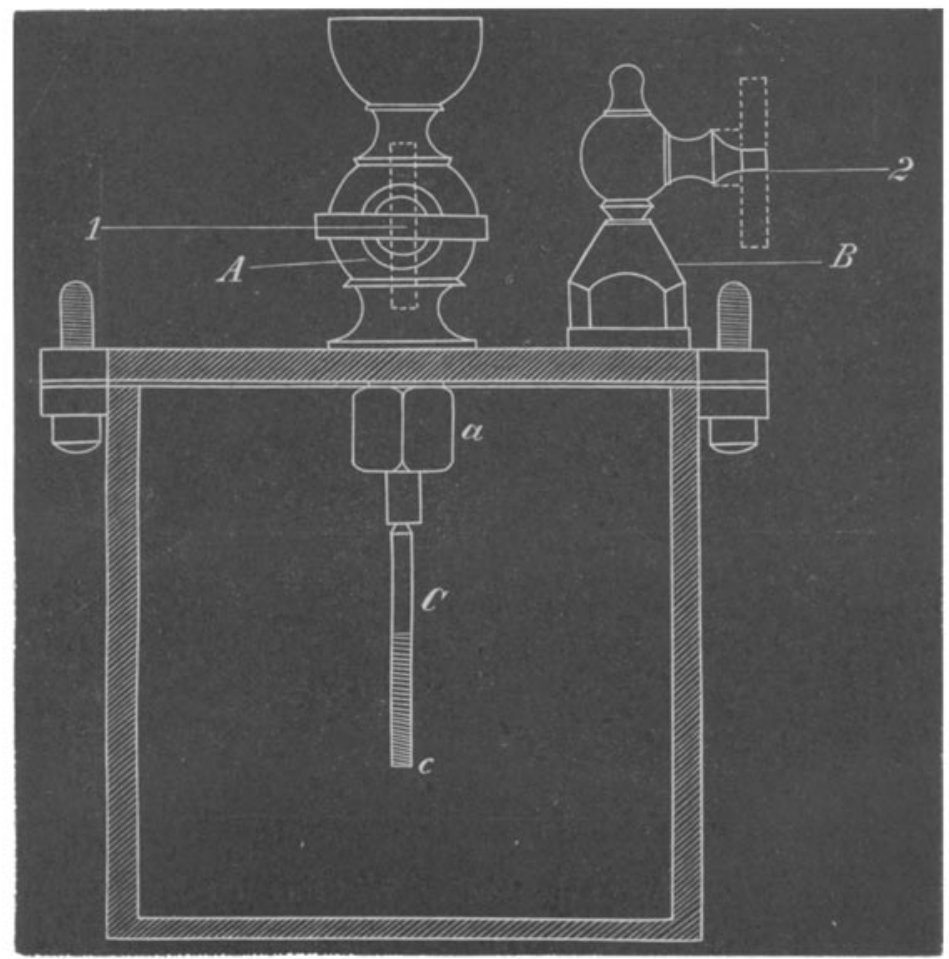

Figur 1.

F'igur 1 .

thoden sind. A m sichersten und vollständigsten erreicht mandieVerdrängung der Luft durch Evacuiren mittelst der Luftpumpe. Zu diesem Zwecke haben wir den folgenden Apparat ${ }^{1}$ ) construirt, welcher unsere oben formulirte Aufgabe in vollkommener Weise erfüllt.

Als Recipient (s. Fig. 1) dient ein $7 \mathrm{Cm}$. hohes und $7 \mathrm{Cm}$.

1) Derselbe wurde ausgeführt von Herrn Mechaniker Petzold, Leipzig, Bayerische Strasse 13. 
im Durchmesser baltendes cylindrisches Hohlgefäss von Messing. Da, wo der Rand des abhebbaren Deckels den Rand des Gefässes beriibrt, ist im ganzen Umkreis eine gut eingefettete Lederscheibe eingelegt. Der Deckel selbst (s. Fig. 2) wird mittelst 6 feiner Schraubenmuttern luftdicht an die Lederscheibe angepresst. An 2 Stellen (s. Fig. 1) trägt der Deckel eine kurze trichterförmige Röhre $A$ und $B$, welche durch ein Schraubengewinde eingefügt sind. Die centrale Röhre A dient zum Eingiessen der Füllungsmasse, während die seitliche B die Verbin-

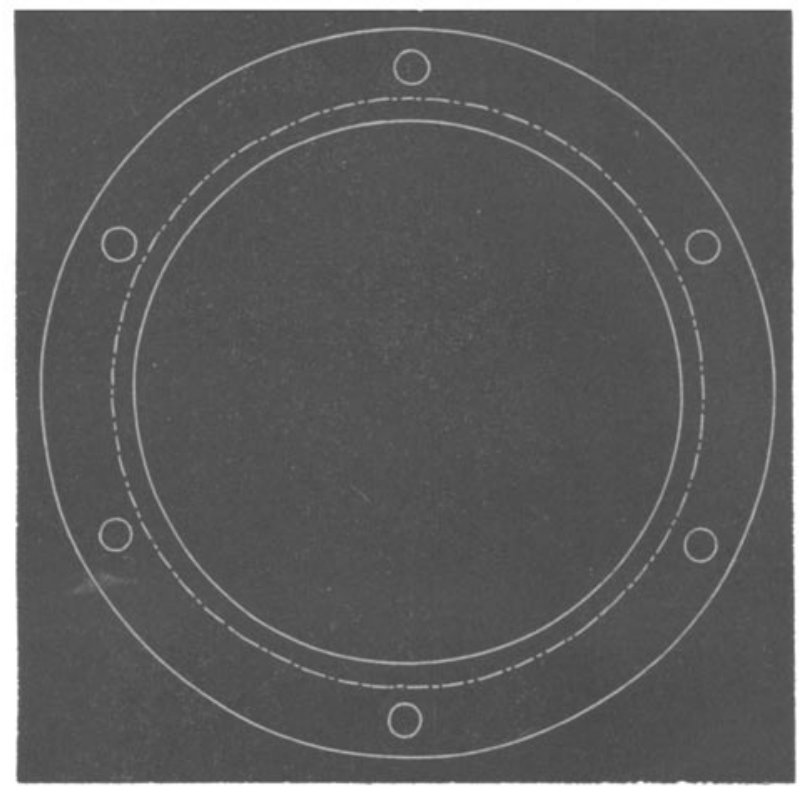

rigur 2.

dung mit der Luftpumpe berstellt. Wie aus der Zeichnung: ersichtlich, sind beide Trichter durch Hähne verschliessbar; letztere sind mit einer Bohrung versehen, und zwar in der Weise, dass, wenn der Hahn in der Stellung 1 steht, also mit dem Trichter einen rechten Winkel bildet, der Trichter geschlossen, wenn der Hahn um $90^{\circ}$ gedreht wird, also in Stellung 2 kommt, geöffnet ist. In das untere Ende von $\mathrm{A}$ bei a wird die Canüle $\mathrm{C}$ gesteckt und mit einer Schraubenmutter befestigt. Diese Caniile vermittelt die Verbindung mit dem zu injicirenden Knochen. Da wir bei unseren Präparaten in der Regel das ovale Fenster als Eingussöffnung benutzen, dieses aber in seinen Durchmessern vielfach variirt, so wird das untere Ende von Ce in verschiedenen, 
den jedesmaligen Durchmessern des ovalen Fensters genau entsprechenden Weiten angefertigt.

Da unsere Absicht auf die Herstellung von Labyrinthpräparaten gerichtet ist, so zerlegen wir das in Wasser grob macerirte Schläfenbein durch einen sagittalen Sägeschnitt in 2 Hälften, Schuppe und äusserer Gehörgang einerseits, Pyramide andererseits. Der obere Bogengang wird auf seiner höchsten Höhe (Eminentia arcuata) etwas angefeilt, die Pyramide fuir einige Stunden in 3 proc. Kalilauge gebracht, um etwaige zurückgebliebene Reste häutiger Substanz zu zerstören, 1 Tag gewässert, dann sorgfältig ansgespritzt, mit Aether entfettet und getrocknet, eventuell rorher mit absolutem Alkohol 24 Stunden lang entwässert. Das runde Fenster wird mit gummirtem Fliesspapier ïberklebt, seine Nische mit Papierbrei, welcher mit dickfliissigem Gummi vermischt ist, ausgefuillt. Ist dieser Brei gut getrocknet, was binnen 1 Stunde durch Trocknen im Luftbad zu erreichen ist, so bildet er einen luftdichten Verschlnss. In das ovale Fenster wird das untere Ende c der Canïle $\mathbf{C}$ gesteckt und mittelst sogenannten Postpacklackes ${ }^{1}$ ) befestigt. Dieser Siegellack hat den Vorzug, dass sein Schmelzpunkt über dem des gleich zu erwähnenden Wood'schen Metalls liegt, also ein Weichwerden desselben während des Durchfliessens des heissen Metalls durch die Canüle nicht zu befürchten ist. Da er zudem einen ausgezeichneten baltbaren Kitt bildet und ein sauberes Arbeiten ermöglicht, so hat er entschieden Vorzuige vor dem porösen Gyps, welcher leicht Metalltheilchen aufnimmt and in welchem die glatte Canüle sich dreht. Kommt es uns auf die naturgetreue Form des ovalen Fensters an, so verstopfen wir auch dieses ebenso und benutzen, wie schon erwähnt, ein Bohrloch im Promontorium als Eingussöffnung. Der innere Gehörgang wird mit angefetteter Watte ausgefüllt, sonst aber auf alle ktinstlichen Stïtzbrücken und Ueberklebungen nach der Art von Bezold, Siebenmann u. A. verzichtet. Hat man sich noch mittelst Durchleitens eines Luftstromes von der ungehinderten Passage im Labyrinth überzeugt, so wird die Canüle C sammt anhängendem Knochen an den Eingusstrichter A angeschraubt, der Recipient und der Hahn des Trichters A geschlossen, während der von B geöffnet wird. Der ganze Apparat wird auf $1 / 2$ Stunde in ein Wasserbad von 50-60 C. gebracht und der Trichter B mit einer Luftpumpe verbunden.

Ursprïnglich benutzten wir die Quecksilberluftpumpe. Allein

1) Derselbe ist zu beziehen von A. de Bous, Hirschberg in Schlesien. 
das Bestreben, das beschriebene Verfahren möglichst zu vereinfachen und Jedem zugänglich zu machen, bestimmte uns, später eine sogenannte Wasserluftpumpe zu benutzen, wie sie in jeder Haushaltung leicht herzustellen ist. Mit ihr erhält man binnen 10 Minuten einen vollkommen ausreichenden Grad von Luftverdünnung. Ist mit der Luftpumpe ein Manometer verbunden, so zeigt die Niveaudifferenz von $25-30 \mathrm{Cm}$. zwischen den Schenkeln desselben diesen ausreichenden Grad von Luftverdünnung an. Nun schliesst man den Hahn der Röhre B, trennt den Recipienten von der Luftpumpe und geht zum Eingiessen der Injectionsmasse utber. Als solche benutzen wir nach Si ebenm ann's Vorgange das von v. Brunn eingeführte Wood'sche Metall. Es besteht aus 4 Theilen Wismuth, 2 Theilen Blei, 1 Theil Zinn, 1 Theil Cadmium und schmilzt bei etwa $60^{\circ} \mathrm{C}$. Das Metall wird in einem gusseisernen Tiegel geschmolzen, der Eingusstrichter A bei geschlossenem Hahn mit fluissigem Metall gefiullt, der Hahn dann langsam geöffnet und Metall beständig nachgegossen. Letzteres strömt unter einem hörbar zischenden Geräusch in den luftverdiunnten Raum. Ist ein genügendes Quantum eingegossen, so wird der Hahn des Trichters B geöffnet, wonach das weitere Einfliessen des Metalls durch das Eintreten der atmosphärischen Luft alsbald aufhört. Man küblt nun den Apparat in Wasser ab, öffnet den Deckel, schraubt das Präparat $a b$ und reinigt die einzelnen Theile des Apparats im Wasserbade von anhaftenden Metalltheilchen, welche sich wieder verwenden lassen. Das Präparat, dessen gelungenen Ausguss man sofort an dem gefüllten oberen Bogengang und gefüllten Aquaeductus vestibuli sieht, wird zur Auflösung des Siegellacks in Alkohol oder Aether gelegt. Um die Canuile vom Präparat zu trennen, wird sie mässig erwärmt, worauf die Trennung leicht vorzunehmen ist.

Zum Zwecke der Corrosion kommt das Präparat in ein mit Fliesspapier gepolstertes flaches Schälchen und dieses in ein mit 20 proc. Kalilauge gefülltes grösseres Gefäss. Nach 8 Tagen wird das Schälchen herausgehoben, ohne das Präparat zu bertihren, gewässert und in frische Lauge gebracht. Trotz wochenlangen Liegens in Kalilauge bleibt die elfenbeinerne Labyrinthkapsel, namentlich um die Schnecke, unlöslich. Deshalb kommt das gewässerte Präparat zum Schluss auf einige Stunden in 5 proc. Salzsäurealkohol. Sollte sich auch hier noch nicht Alles lösen, so wird nochmals einige Tage mit Kalilauge macerirt, dann das Präparat gewässert und getrocknet. 
Methode zur Gewinnung von Corrosionspräparaten des Ohrlabyrinths. 203

Mittelst des beschriebenen Verfahrens erhält man nicht nur einen Ausguss der drei Bogengänge und der Schnecke, sondern es kommen auch zur Anschauung die naturgetreue Form des ovalen und runden Fensters, der Saceuli, der Ampullen, der Verlauf der Aquäducte sammt Nebenkanälen, des Nervus cochleae ef vestibuli.

Es ist mir eine angenehme Pflicht, Herrn Prof. Ludwig fuir seine Unterstittzung bei meiner Arbeit meinen Dank auszusprechen. 